Devel opment of Car di ac Rhythms in Altricial Avi an Enbryos

\begin{tabular}{|c|c|}
\hline $\begin{array}{l}\text { 弚の他 (別言語等) } \\
\text { のタイトル }\end{array}$ & 晚成離栄性鳥類胚における心拍リズムの発垟 \\
\hline 著者 & PEARSON James, TAZAMA Hi roshi \\
\hline $\begin{array}{l}\text { jour nal or } \\
\text { publ i cati on title }\end{array}$ & Menoi rs of the Mur or an Institute of Technol ogy \\
\hline vol une & 49 \\
\hline page $r$ ange & $9-18$ \\
\hline year & $1999-11-30$ \\
\hline URL & ht t p: //hdl . handl e. net /10258/149 \\
\hline
\end{tabular}




\title{
Development of Cardiac Rhythms in Altricial Avian Embryos
}

\author{
James T. PEARSON* and Hiroshi TAZAWA*
}

(Accepted 31 August 1999)

\begin{abstract}
Mean heart rate (MHR) was determined during incubation and in hatchlings of 14 altricial avian species to investigate 1) if there is a comon developmental pattern of hedrt rate in altricial embryos and 2) if heart rate changes during ineubation are correlated with changes in embryonic growth rate. On the basis of normalized incubation MaR increased approximately linearly in 12 of 14 species from as early as $30-40$ of of incubation to that of pipped embryos. The MHR of hatchlings was equal to or higher than that of pipper embryos in 7 species. Passerine embryos and hatchlings maintained higher MHR in comparison to parrots of similar egg trass, which may reflect phylogenetic differences in development. Embryonic Mer increased at a higher rate while embryonic growth rates were highest during the first 40 \% of incubation in tit, budgerigar and crow ambryos than during subsequent development when relative growth rates decreased. MHR became independent of yolk-free wet mass at a smallex fraction of hatchling mass in budgerigar and crow than in the tit, suggesting that MAR is more likely to increase continuously after 408 of incubation in small altricial species than larger species.
\end{abstract}

Keywords: Altricial, Ballistocerdiogram, Electrocardiogram, Embryo, Growth rate, Hatchling, Mean Heart Rate

\section{INTRODUCTION}

Altriciality in avian embryos is considered to have evolved by hatching significantly earliex in the developmental sequence, resulting in a shortening of the incubation period in comparison to precocial embryos ${ }^{i 6,2 \mathrm{E})}$. In general, the physiological state of the altricial hatchling is less mature than that of the precocial hatchling from eggs of similar mass ${ }^{(25,28)}$. Comparisons of developmental changes in embryos across the altricial-precocial continuura are essentially limited to measurements of metabolism, growth efficiency and incubation water budgets ${ }^{13,5,6,10}$, 15,27,28). Typically the metabolism of altricial embryos increases at a slower rate and reaches a lower maximal rate at hatching than precocial species of similar egg mass(27) * These differences likely reflect the lower costs of maintaining the more immature tissues of the altricial embryo duxing its development ${ }^{14}$. Therefore it is reasonable to predict that the developmen differ across the altricial-precocial continuum.

The eggs of most altricial species are typically a magnitude smaller than most precocial and semi-precocial species. To date, the development of embryonic mean heart rate (MHR) has been extensively studied in precocial species ${ }^{(1,9,13,16,17,19,21,22,26)}$, but has only been studied in three species of semiprecocial seabird(20,25) and two species of altricial bird ${ }^{(8,24)}$. Non-invasive embryonic MHR measurements in small avian eggs have recently become possible ${ }^{(13)}$, and we wished to apply these techniques to study a range of altricial species. However, measurement of embryonic HR during the early stages of incubation by this non-invasive method, the ballistocardiogram (BCG), is not possible because of the low signal to noise ratio. Nevertheles's, several studies of precocial embryos suggest that MHR changes greatly over the entire incubation period. In the chicken (Gallus domesticus) and the king quail (Coturnix chinessis), three phases in HR development are clearly recognizable during incubation ${ }^{(1,9,13,26)}$. After the heart beats for the first time, a periodic beat develops and is initially low, but MHR rapidly increases over the early phase. The data of several studies show a second (intermediate) phase, from about 408 of incubation, when MHR

\footnotetext{
Department of Electrical and Electronic Engineering
} 
increases at a reduced rate in some precocial species or is stable for a period in other species ${ }^{(1,9,13,19,26)}$. Some of these species decrease HR significantly at the end of the second phase, immediately prior to internal pipping of the aircell ${ }^{(13,17,19\}}$, but the cause remains unclear and in the final phase (after 858 of incubation) HR increases again. In contrast, the HR of embryos of all three semi-precocial species investigated so far is stable during late incubation until pipping, when likewise HR increases ${ }^{(20,25)}$. Thus the development of MHR appears to be very variable among precocial species during the intermediate and final phases. Absolute growth rates of precocial Galliform embryos increase exponentially from about 608 of incubation to hatching, following the period of complete development of the extra-embryonic membranes ${ }^{(10)}$. As the described incxease in embryonic MHR of precocial embryos precedes the significant increases in maintenance costs after $60 \%$ of incubation ${ }^{(10)}$, these observations suggest that MHR increases independent of embryonic metabolism.

Alternatively, we hypothesize that the development of MHR during incubation in both precocial and altricial embryos is directly related to embryonic growth rate, and that phase changes in MHR are correlated with a reduction in growth rate during incubation. Therefore our primary aim was to investigate the developmental patterns of HR in 14 species of altricial embryos. Using the ballistocardiogram method $(9,13,16,21,22)$ we noninvasively investigated in experiment I the development of MHR in embryos during the last $60 \%$ of incubation in 10 passerine and 2 parrot species, over a range of fresh egg masses between 1-6 g. In experiment II we semiinvasively determined the development of MHR in 5 altricial species (egg masses $1.5-21$ g) throughout incubation, and its relationships with embryonic growth. We used an electrocaxdiogram (ECG) method in experiment II to determine MHR, since this method can detect the cardiogenic signals of the earliest embryos. The development patterns of MHR in these altricial species were then compared with available data from precocial species ${ }^{1,}$ $9,13,26)$ on the basis of normalized incubation time.

\section{MATERIALS AND METHODS}

\subsection{Experiment I}

Eggs from the following passerine species were collected under permit from the Japanese Department of Environment during March-August of 1997 and March-May of 1998, from the surrounds of Muroran and Noboribetsu Cities; great tit (Parus major), marsh tit (parus palustris), varied tit (Parus varius), Japanese tree sparrow (Passer montanus), house martin (Delichon urbica), Japanese bunting (Emberiza spodocephala), red-cheeked myna
(Sturnus philippensis), and brown-eared bulbul (Hypsipetes amaurotis). Eggs of domesticated altricial species, of known incubation age, were obtained from adult breeding birds, which were kept in a laboratory. This included two parrot species, the budgerigar (Melopsittacus undulatus) and the cockatiel (Nymphicus hollandicus), and two passerine species, the zebra finch (Taeniopygia guttata) and the Bengalese finch (Lonchura striata var. domestica). Eggs of wild species were brought to the laboratory and incubated together with that of domesticated species in a small still aix incubator (zenkei table top model 40 , Japan) at $38^{\circ} \mathrm{C}\left( \pm 0.2^{\circ} \mathrm{C}\right)$. Relative humidity was controlled by vents so that eggs achieved approximately $15 \%$ mass loss duxing incubation (RH 55-65\%). All HR measurements were made in a larger still-air incubator (Sakura IF-B3, Tokyo) at $38^{\circ} \mathrm{C}( \pm 0.2$ $\left.{ }^{\circ} \mathrm{C}\right)$. During incubation eggs were turned $180^{\circ}$ by hand every two hours between $08: 30$ and $18: 30$ until hatching.

\subsection{Experiment II}

MHR was determined for embryos of the great and marsh tits, budgerigar, and the carrion and jungle crows (Passeriformes, Corvus corone and c. macrorhynchos). Budgerigar eggs were measured for fresh egg mass on the day of lay, numbered for identification and then returned to the nest for parental incubation until the desired incubation day when the eggs were collected again for $\mathrm{HR}$ measurements. In contrast, tit and crow nests in the city of Muroran, Hokkaido, Japan, were monitored (inspections every $2-3$ days) to determine laying dates and the commencement of incubation. Eggs were then collected and incubated in an artificial incubator maintained at $36^{\circ} \mathrm{C}$ for crow eggs and $38^{\circ} \mathrm{C}$ for tit eggs. Tit and crow eggs were turned until the day of final HR measurements, after which embryos were sacrificed to determine embryo mass. Typically, 4-5 eggs from each tit clutch (clutch range 7-12 eggs) were collected on a desired incubation day and measured the same day. As fewer crow and budgerigar eggs were available for experiment II several embryos of these species were measured for HR over several consecutive days to confirm the developmental patterns of individual embryos before being sacrificed after the final measurement. A limited number of hatchlings from eggs used in experiment $I$ were measured for $M H R$ and then sacrificed to determine yolk-free wet mass ( $n$ $=5$ and 4 for tits and crows, respectively).

\subsection{Heart rate measurement}

2.3.1 Ballistocardiogram of embryos in experiment I. An egg was placed on a ceramic plate, which was supported on a floating platform within the experimental incubator. The stylus of an audiocartridge was brought into contact with the surface of the egg and the ballistocardiogram (BCG) was measured to 
determine MHR (defined here as $M_{B R}$ ) as described in previous studies of this laboratory ${ }^{(13,16,21,22 i}$. MHR BeG was then calculated using Burg's algorithm (power spectrum of each $5-s$ HR interval) according to pearson et al. ${ }^{(13)}$ and averaged over all 5-s HRs for each 10-min period for all embryos of that species on each day of incubation. Only one embryo was placed in the experimental incubator and measured at a time, so measurements were made sequentially on each embryo after a 45-min temperature equilibration period. Thus each embryo required a total of $55 \mathrm{~min}$. The equilibrium period was necessary not only to compensate for cooling of the egg and incubator, but also was considered to reduce the possible effects of recent handling of each egg. Measurements were completely non-invasive, and were made each day of incubation until hatching.

2.3.2 Electrocardiogram of embryos in experiment II. Three $30 \mathrm{~mm}$ long copper wires $(0.1 \mathrm{~mm}$ D) were used as ECG leads. Each wire was bent at right angles $2-4$ min from one end (length dependent on size of egg), which was inserted into a hole made on the upper surface of the egg by carefully puncturing the eggshell and shell membranes with a 25-gauge hypodermic needle sterilized in alcohol. The three electrodes were inserted to form an equilateral triangle with sides $10 \mathrm{~mm}$ long in the tit and budgerigar and $25 \mathrm{~mm}$ long in the crow. Epoxy glue was used to seal the holes and fix the electrodes in place with minimal reduction of the diffusive surface area of the egg. Prepared eggs were rewarmed in the small incubator until the epoxy hardened ( $1 \mathrm{~h}$ ) before transfer to the measurement incubator. The electrical signal was amplified, band-pass filtered before being digitized and recorded on a personal computer. Bandpass filtex frequencies varied with both embryonic age and the quality of the signal. Embryos during the first $70 \%$ of incubation wexe usually filtered between $2-10 \mathrm{~Hz}$ and more mature embryos between 30-120 $\mathrm{Hz}$. ECG signals were recorded for 30-min periods for each embryo after allowing eggs to reach thermal. equilibrium ( $45 \mathrm{~min}$ ) . Digitized signals were recorded on a personal computer at a sampling frequency of $100 \mathrm{~Hz}$ for embryos (< $70 \%$ of incubation). Later these signals were30-min interval was calculated by a Fast Fourier transform using the reconstructed signals to determine the MHR of each embryo.

2.3.3 pipped embryos and hatchlings. The MHR of hatchlings of all species, except the crows, were measured using a modification of a non-invasive method ${ }^{(\infty)}{ }^{18,23)}$ as described in pearson et al. ${ }^{133}$ for king quail. Individual hatchlings were placed in a resting position on a piezo-electric film within a glass petri dish inside the same incubator as used for embryonic measurements. The film signal was then amplified, filtered $(5-24 \mathrm{~Hz})$ and sampled as for the BCG measurements of embryos. The large pipped embryos and hatchlings of crows were measured by ECG as the QRS complex were more easily recognizable above background and external noises. In this case, instantaneous HR was determined from the time interval between consecutive $R$ waves using a threshold method and a sampling frequency of $4000 \mathrm{~Hz}$. Non-invasive, flexible $\mathrm{Ag} / \mathrm{AgCl}$ gel $\mathrm{ECG}$ electrodes (Vitrode A50, Nihon-Kohden) were used for crow hatchlings as described for quail and chickens ${ }^{(11,13)}$. ECG signals from pipped embryos and crow hatchlings were amplified

Table 1. Mean fresh egg masses (M) and sample sizes ( $N$ ) of avian species used to deternine embryonic and hatchiing mean heart rate in experiment $I$. Numeral ID indicates a unique number used to identify each species in figures $1-2$.

\begin{tabular}{|c|c|c|c|}
\hline Species & Numeral ID & $n$ & Mean $M(g)$ \\
\hline Zebra finch Taeniopygia guttata & 1 & 5 & 0.97 \\
\hline Bengalese finch Lonchura striata var. domestica & 2 & 5 & 1.10 \\
\hline Tit species combined, 3 Parus spp. & 3 & 21 & 1.55 \\
\hline Tree sparrow Passer montanus & 4 & 11 & 2.09 \\
\hline House martin Delichon urbica & 5 & 5 & 2.25 \\
\hline Japanese bunting Emberiza spodocephala & 6 & 2 & 2.60 \\
\hline Red-cheeked myna sturnus philippensis & 7 & 2 & 4.14 \\
\hline Brown-eared bulbul_Hypsipetes amaurotis & 8 & 3 & 6.40 \\
\hline Budgerigar Melopsittacus undulatus & 9 & 13 & 2.20 \\
\hline Cockatiel Nymphicus hollandicus & 10 & 7 & 5.10 \\
\hline
\end{tabular}


and filtered between $50-150 \mathrm{~Hz}$ and the MHR determined by averaging the instantaneous HRs over $30-m i n$ intervals.

\subsection{Embryonic growth and statistics}

Eggs from experiment II were opened after HR measurements, soon after cooling the eggs to $10^{\circ} \mathrm{C}$. Embryos were removed, blotted dry with tissue paper, excepting embryos younger than 30 of the incubation period, which were too fragile to be directly blotted with tissue paper. Embryos were then weighed to $0.1 \mathrm{mg}$ on an electronic balance (AND model ER-180A). Internal yolk reserves were removed from externally pipped embryos and hatchlings and therefore only yolk-free embryo mass (denoted as $\left.M_{y t}(g)\right)$ was included in analyses.

Linear regressions between variables ( $\log -\log$ or semi-log) were fitted by the method of least squares using sxstari29) or by a personal program after yeager and ultsch ${ }^{(30)}$ to determine biphasic linear regressions as appropriate. A coefficient of determination $\left(r^{2}\right)$ is presented for significant relations. Fresh egg mass was not known for eggs of some species, collected during mid- or lateincubation, but was estimated from egg length $(L)$ and maximum width $(W)$ at the equator of the egg according to the following relation, fresh egg mass $=a(L W)^{2}+b$, where $a$ and $b$ are constants determined for each species by linear regressions fitted to data of eggs of known fresh egg mass.

\section{RESULTS}

\subsection{Experiment I}

The embryonic $\mathrm{MHR}_{\mathrm{ecc}}$ of 12 altricial species (fresh egg mass range 0.97-6.40 g) is presented in relation to normalized incubation time (percentage of total incubation) in figure 1. Mean fresh egg mass was significantly different between tit species (mean egg mass: $P$. major $1.59 \mathrm{~g}(n=12)$, $P$. palustris $1.39 \mathrm{~g}$ (6), $P$. varius $1.68 \mathrm{~g} \mathrm{(3),} \mathrm{ANOVA} F_{2,1 \mathrm{E}}=7.211$ $p<0.005)$. However, the developmental patterns of MHR $_{\text {Ecq }}$ were combined and averaged for the three tit species examined. Embryos of the Japanese bunting, red-cheeked myna and brown-eared bulbul were already well developed (55-70\% of incubation) when the eggs were collected and first measured. In contrast, the eggs of the other species were collected in early incubation stages and so the first $\mathrm{MHR}_{B C G}$ measurements in figure 1 represent the age when HR signals were first detectable by this method. The $100 \%$ value indicates the MHR of hatchlings (determined by piezo-electric film) for all 12 species. In all species there was a trend for $M_{H R}$ to increase continuously with incubation time, except for cockatiel embryos, which decreased MHR $_{B=c}$ until pipping. Furthermore, $\mathrm{MHR}_{B C a}$ increased at a higher rate after $80-95 \%$ of incubation than during the period before in all species, including the cockatiel. In general $\mathrm{MHR}_{\mathrm{Bco}}$ increased with decreasing fresh egg mass, when compared between species at any given point during incubation prior to internal pipping (90-95\% of incubation), but was more variable in level of $\mathrm{MHR}_{\mathrm{BaG}}$ between species after internal pipping. Embryonic MHR $_{E C G}$ was maximal at 90-95\% of incubation, ranging between $370-450$ bpm, but was only higher than that of resting hatchlings in 5 of the 12 species, including the three tit species (Fig. I).

In figure 2 the developmental patterns of embryonic $M_{H C}$ in two paired-comparisons of parrots and passerines are illustrated for species with similar fresh egg masses. In common to both cases the parrot and passerine species increased $M R_{B C G}$ most during the final pipping phase (85-100\% of incubation) + However, $M_{H R} R_{B C G}$ continued to increase in the tree sparrow and brown-eared bulbul embryos during midincubation, whereas $\mathrm{MHR}_{\mathrm{BCG}}$ changed little in budgerigar and decreased in cockatiel embryos during mid-incubation. At any one time during incubation the passerines maintained higher $\mathrm{MHR}_{B C G}$ than the parrots.

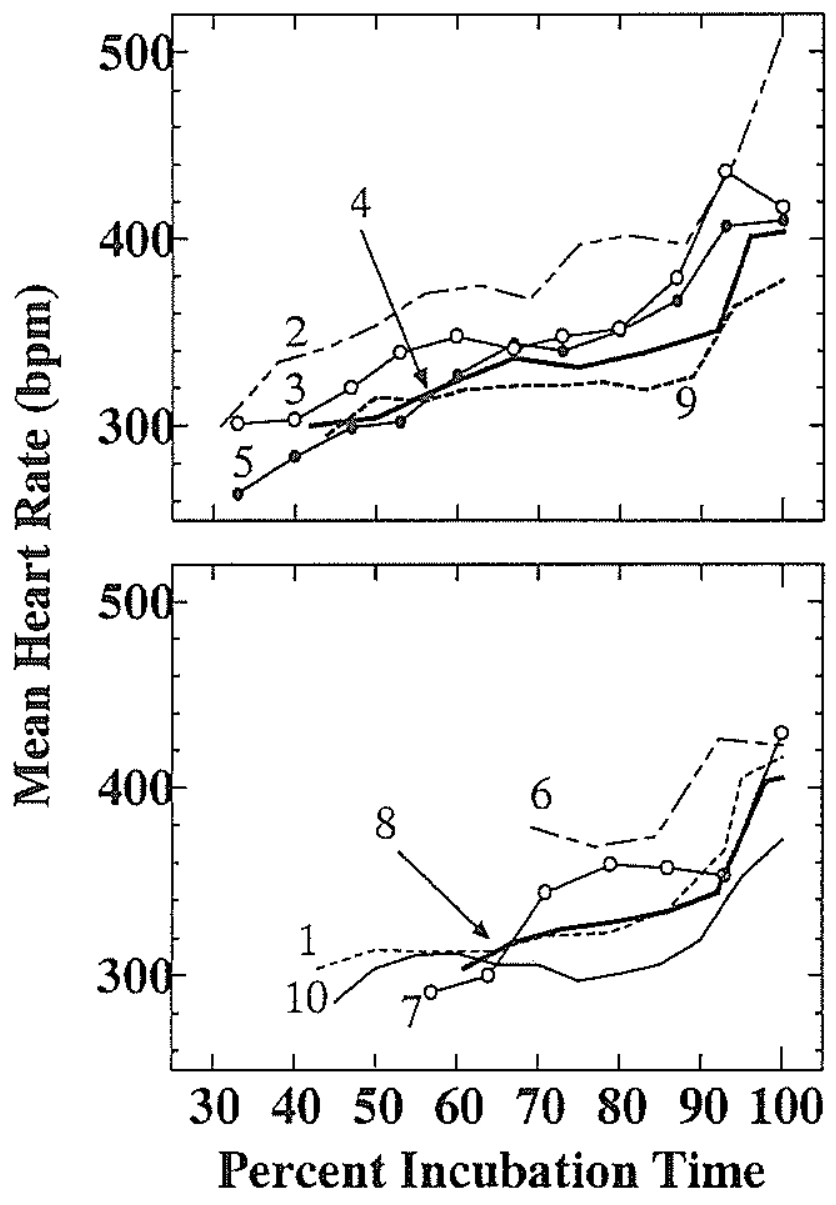

Fig. 1. Embryonic mean heart rate (bpm) of 12 altricial avian species during normalized incubation (percent of total incubation) determined by ballistocardiogram. Final point indicates the MHR of hatchlings. Numerals indicate species as listed in Table 1 . 


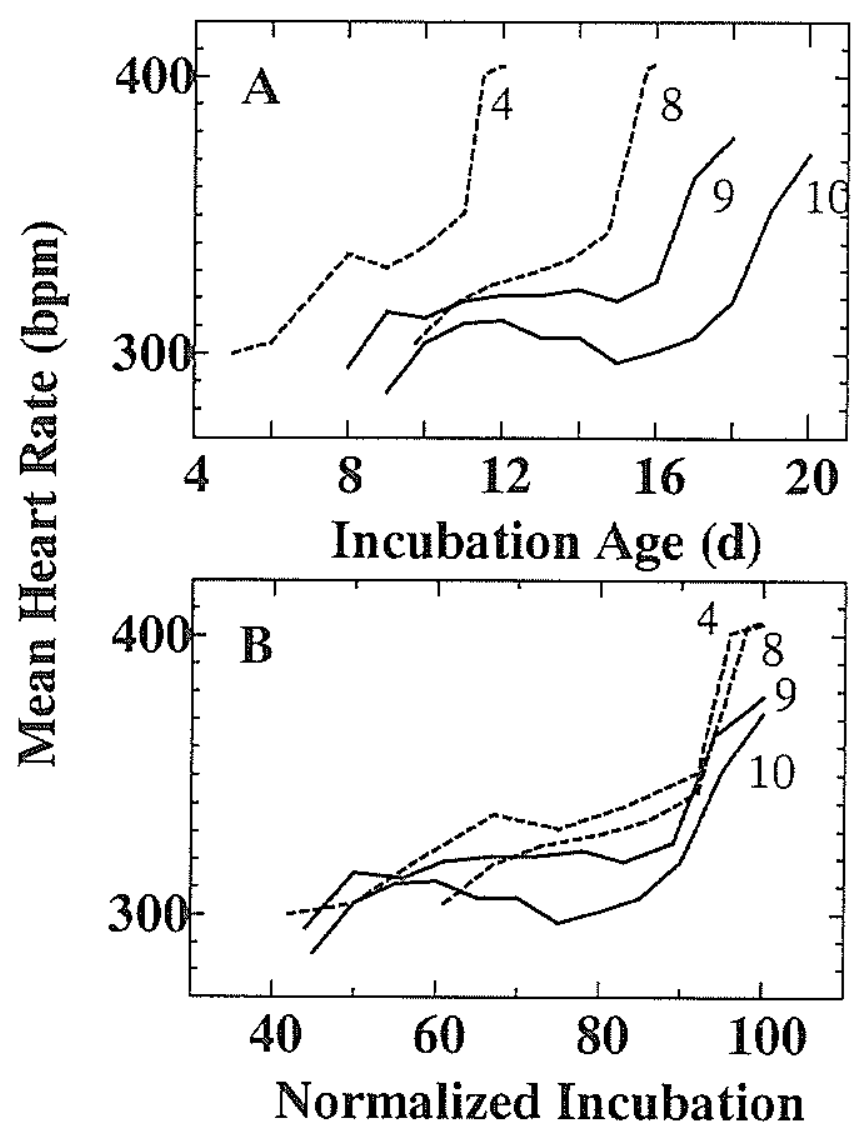

Fig. 2. Comparison of the development of mean heart rate (bpm) in relation to incubation day (A) and normalized incubation (B) for passerine-parrot species pairs of fresh egg mass $2 \mathrm{~g}$, Japanese sparrow (line 4) and budgerigar (9), and 5-6 g, brown-eared bulbul (8) and cockatiel (10).

\subsection{Experiment II}

Figure 3 illustrates the MHR $_{\text {Ecs }}$ of individual tit, budgerigar and crow embryos and hatchlings in relation to incubation day. Five clutches of great tit and one clutch of marsh tit eggs were collected (mean fresh egg mass $=1.51 \pm 0.09 \mathrm{~g} \mathrm{SD}, n=34)$ and MHR was determined from day 3 of incubation up until hatching and for 5 hatchlings (Fig. 3A). MHR of tits determined by ECG was similar to that determined by BCG, and in both experiments MHR increased continuously with incubation age from $170 \mathrm{bpn}$ to $400 \mathrm{bpm}$. Budgexigar eggs were collected for ECG measurements from successive clutches of two females only (mean fresh egg mass $=2.18 \pm 0.14 \mathrm{~g} \mathrm{SD}, n=20$ ). Embryonic budgerigar $\mathrm{MHR}_{z c \sigma}$ increased continuously between 4 and 8 days of incubation from 170 bpm to 290-340 bpm (Fig. 3B). From day 8 both $\mathrm{MHR}_{E c a}$ and $M H R_{a c c}$ were stable, but variable between individual embryos, at an average of $310 \mathrm{bpm}$ until day 16 , one day prior to internal pipping. Eggs from one jungle and 6 carrion crow clutches were used for ECG measurements (mean fresh egg mass $=20.84 \pm 1.56 \mathrm{~g} \mathrm{SD}, n=20$ ). The three jungle crow eggs were pipped (day 19) at the time of collection and were measured from the same day until hatching, after which they were

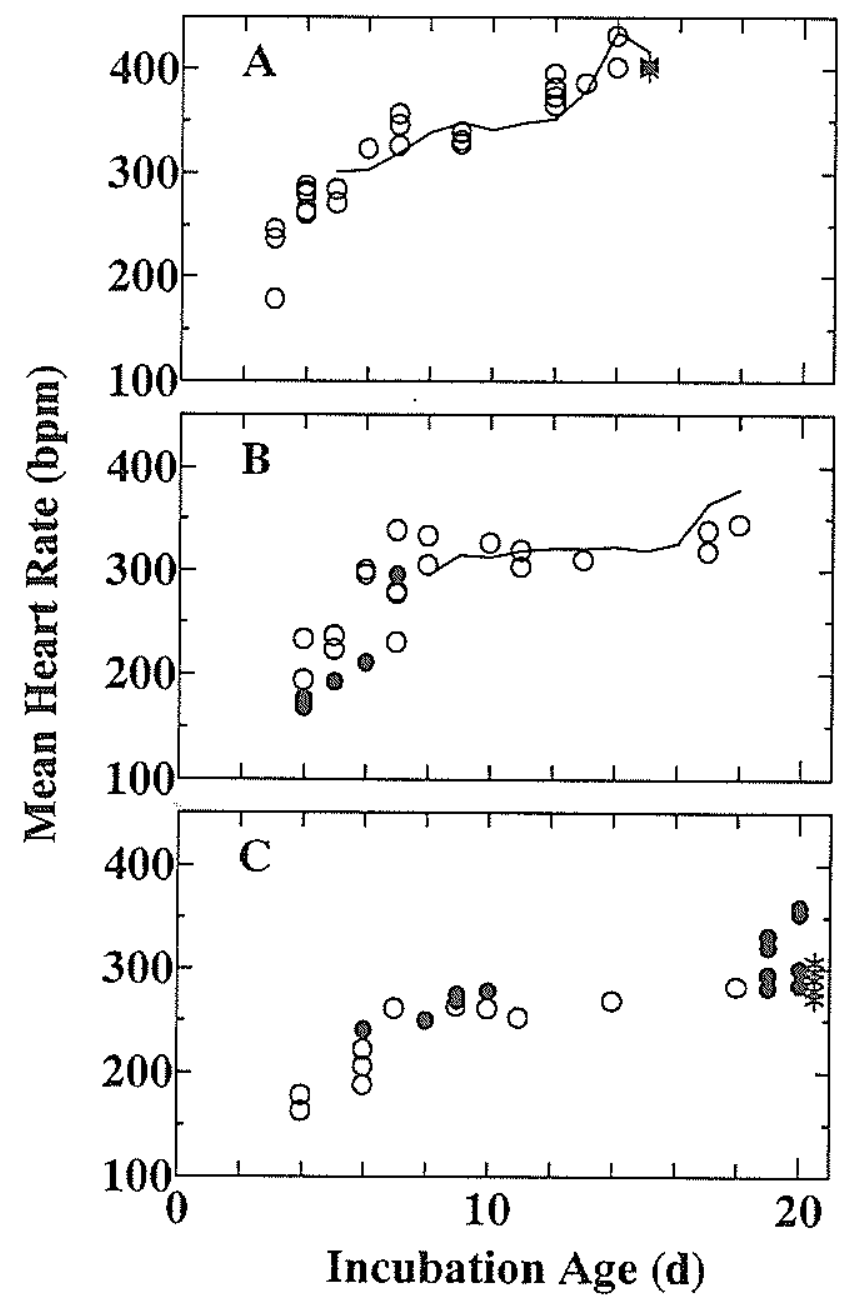

Fig. 3. Mean heart rate (bpm) of embryos and hatchlings of tits (A), budgerigars (B) and crows (C) in relation to incubation age (d). Solid ifnes indicate the daily mean heart rate in tits and budgerigars determined by ballistocardiogram for embryos and by piezo-electric film for hatchlings (A $\& \mathrm{~B}$; as in Fig. 1). Open circles: MHR determined by ECG of embryos sacrificed for mass determinations ( $A$ : tits $n=29$; $B$ : budgerigars $n=18$; C: crows $n=12$ ); filled circles: MHR determined by ECG for some of the same embryos as the open circles before being sacrificed; asterisks; MHR determined by ECG of hatchlings (A: tits $n=5$; C: crows $n=4$ ).

sacrificed together with one carrion crow hatchling to determine $\mathrm{M}_{\mathrm{yf}}$. Crow embryonic $M_{\text {MEs }}$ development (both species combined) was similar in pattern to that of budgerigar

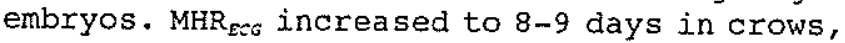
and then changed little during the remainder of incubation at 270-300 bpm, except during pipping when $M_{H R} R_{E c}$ increased to $330-360 \mathrm{bpm}$, but decreased again after hatching to about 300 bpm (Fig. 3C).

The MHR $_{g c s}$ of embryos is presented in relation to increasing $M_{y f}$ of embryos and hatchlings in figure 4 . In the case of the budgerigar, hatchling $M_{y f}$ was not measured, but calculated to be $1.22 \mathrm{~g}$ from the average mass of 7 hatchlings (experiment I) minus $0.08 \mathrm{~g}$ yolk, assuming yolk is 6 \& of egg mass ${ }^{(7)}$. The MHR 


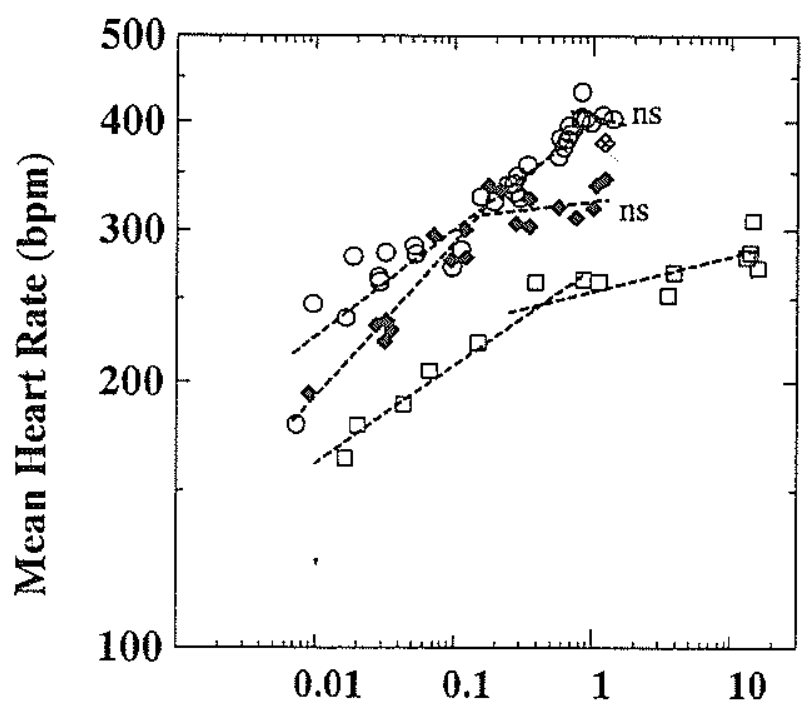

Yolk-free wet mass $(\mathrm{g})$

Fig. 4. Relationships between the logarithm of mean heart rate (bpm) and yolk-free wet mass ( $g$ ) in tits (circles), budgerigar (diamonds), and crows (squares). Individual embryos and hatchlings same as in Figure 3. Lines indicate significant biphasic linear regressions $(P<0.05)$ listed in Table 2 , except where noted by $n s$, which indicates a nonsignificant regression (regression slope n.s. from zero). Crossed-diamond: MHR of budgerigar hatchlings based on estimated Myt

relationship with embryo mass clearly showed two phases in all species tested. During the early phase $\mathrm{MHR}_{x c c}$ was directly related to $\mathrm{M}_{y f}$, but was independent of $M_{y f}$ in the budgerigar above $0.16 \mathrm{~g}$, and $0.3 \mathrm{~g}$ in the crow, but $\mathrm{MHR}_{\mathrm{Ees}}$ did not become independent of $M_{y:}$ in tit embryos until $0.8 \mathrm{~g}$. Thus the phase change of these relations with $\mathrm{MHR}_{\mathrm{ECC}}$ in tit, budgerigar and crow embryos was reached at $80 \%, 13 \%$ and $2 \%$ of their respective hatchling $M_{y t}$. The slope of regressions (log-log) fitted to $M H R_{E c c}$ on $M_{Y z}$ relations during the first phase of development was significantly higher in the tits and budgerigar than the crows (Table 2 ). However, there was significant overlap in the 95\% confidence intervals of the regression slopes between the tits and budgerigar. On the basis of normalized incubation time the phase change in $M_{E R}$ occurred at about 38-40\% of incubation in the species tested (Fig. 5 and Table 3). The logarithm of embryo $M_{y}$ increased approximately linearly with normalized incubation time at a higher rate during the first 45-508 of incubation than during the subsequent period (Fig. 6).

\section{DISCUSSION}

4.1 Developmental patterns of heart rate in altricial embryos

In this study we were able to measure the mean heart rate (MHR) of altricial avian embryos and their hatchlings non-invasively, across a range of egg masses, $0.97-6.40 \mathrm{~g}$. Using the ballistocardiogram method we have determined the developmental patterns of MHR from as early as 30-408 of incubation. However, cardiac contractions are not sufficiently strong to be discerned from the noise in the signals in the period before our first measurements by the BCG method. The patterns iliustrated in figure 1 on the basis of normalized incubation period and two previous studies of altricial species ${ }^{(8,24)}$ indicate that there is some diversity in developmental patterns. All the species examined show that MHR increases in two phases after $40 \%$ of incubation. In common to these developmental patterns was a final phase of rapid MHR increases after 85-958 of incubation, when embryo activities increase in association with the pipping of the shell membranes and hatching. However, the development of MHR is most variable between altricial species in the intermediate phase, 40-85s of incubation. The results presented here also indicate that phylogenetic constraints probably influence MHR developmental patterns. The MHR of

Table 2, Relationships between mean heart rate (MHR, bpm) and yolk-free embryo mass (Myf, g). Regression equations ( $\left.\log M H R=a+b \cdot \log \mathrm{M}_{y^{f}}\right)$ indicate significant biphasic relations $(P<0.05)$ determined by the method of Yeager and Ultsch (1989). Otherwise regression slope $n .5$. Erom zero. Phases $I$ and II indicate regressions below and above the inflection point, respectively.

\begin{tabular}{|c|c|c|c|c|}
\hline Species & $\begin{array}{l}\text { Inflection point } \\
\left(\mathrm{M}_{\mathrm{yf}}, \mathrm{g}\right)\end{array}$ & Log $a$ & $b \pm S E$ & $r^{2}$ \\
\hline Tit & 0.80 & $I: 2.601$ & $0.124 \pm 0.009$ & 0.887 \\
\hline & \multicolumn{4}{|c|}{ II: n.s. } \\
\hline \multirow[t]{2}{*}{ Budgerigar } & 0.16 & $I: 2.641$ & $0.179 \pm 0.016$ & 0.965 \\
\hline & \multicolumn{4}{|c|}{ II: $n .5}$. \\
\hline \multirow[t]{2}{*}{ Crow } & 0.30 & I: 2.434 & $0.113 \pm 0.010$ & 0.977 \\
\hline & & $I I: 2.406$ & $0.040 \pm 0.040$ & 0.807 \\
\hline
\end{tabular}


Table 3. Relationships between mean beart rate (MHR, bpm) and normalized incubation time ( $I$, 8 ) . Regression equations $(\log M H R=a+b I)$ indicate significant biphasic relations ( $2<0.05)$ determined by the method of Yeager and Ultsch (1989). Phases I and II indicate regressions below and above the inflection point, respectively.

\begin{tabular}{lcccc}
\hline Species & $\begin{array}{c}\text { Inflection point } \\
(I, 8)\end{array}$ & Log $a$ & $b \pm S E$ & $x^{2}$ \\
\hline Tit & 40 & I: 2.240 & $0.0064 \pm 0.0016$ & 0.719 \\
& & II: 2.439 & $0.0017 \pm 0.0002$ & 0.797 \\
Budgerigax & 38 & I: 2.124 & $0.0090 \pm 0.0020$ & 0.703 \\
& 38 & II: 2.456 & $0.0007 \pm 0.0004$ & 0.358 \\
Crow & I: 2.014 & $0.0100 \pm 0.0030$ & 0.780 \\
& & II: 2.374 & $0.0008 \pm 0.0003$ & 0.582 \\
\hline
\end{tabular}

passerine embryos increases to higher levels throughout the incubation period than that of parrots of similar fresh egg mass, and do so within significantly shorter incubation periods (Fig. 2). The low growth rates (embryonic and posthatching) and low metabolic intensities of parrot embryos are correlated with developmental times that are significantly longer than that of passerines ${ }^{6 .}$ 7,121 . Thus the lower metabolic demands of parrot embryos at any one time are correlated with the only developmental patterns that exhibited stable MHR or significant decreases in MHR in the case of the cockatiel, during the intermediate phase of incubation.

ECG measurements confirm that in fact the development of MHR is best described by three phases (early, intermediate and final) in relation to incubation time in altricial species over the whole incubation period (Fig. 3). After the embryonic heart beats for the first time and becomes periodic MHR increases according to significant multiphase linear relations with respect to normalized incubation. During the early phase, 20-40\% of incubation, MHR increases at a higher rate than during the intermediate phase, 40-85\% of incubation (Fig. 5). A similar phase change at about $40 \%$ of incubation can be demonstrated in the MHR of precocial chicken and king quail embryos ${ }^{(1,9,13,26)}$. Therefore we suggest that there may not be significant differences in the development of MHR in altricial and precocial. embryos during early development based on this limited data. However, MHR in altricial species in general increases continuously during the intermediate phase and shows larger increases still during the final (pipping) phase. In contrast, the developmental rates of MHR in precocial species are more variable in pattern (positive and/or negative changes) and the extent of absolute increases during both the intermediate and final phases of incubation ${ }^{300}$ rat. 19). Much of the differences in developmental patterns of avian embryos may relate to fresh

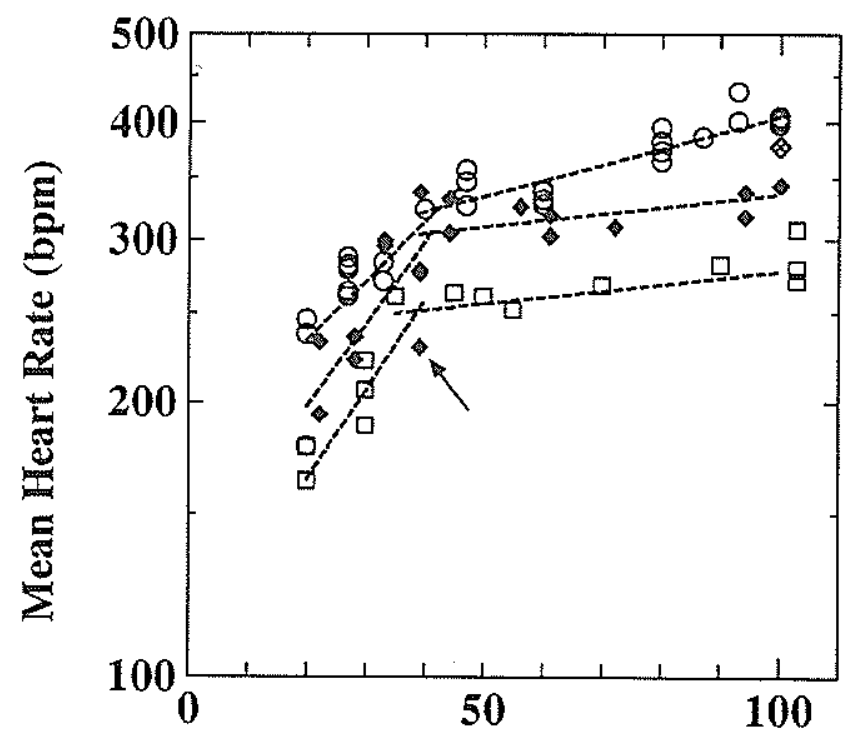

\section{Normalized Incubation}

Fig. 5. Relationships between the logarithm of mean heart rate (bpm) and normalized incubation time (s) in tits (circles), budgerigar (diamonds), and crows (squares). Lines indicate significant biphasic linear regressions listed in Table 3. Arrow: indicates one outlier excluded from analysis of budgerigar data; crossed-diamond: MHR of budgerigar hatehlings based on estimated Mys.

egg mass ${ }^{(4)}$, but further investigations of larger altricial and semi-altricial species are required to determine the points of commonality and differences in relation to developmental mode of birds.

\subsection{Relations between heart rate and embryonic} growth

Relative growth rates of embryos are highest during the first 40 of incubation in both the precocial Japanese quail (Coturnix coturnix) and a variety of altricial species ${ }^{(7)}$. Similarly, the rate of increase in embryonic $M_{y f}$ of altricial tits, budgerigar and crows 


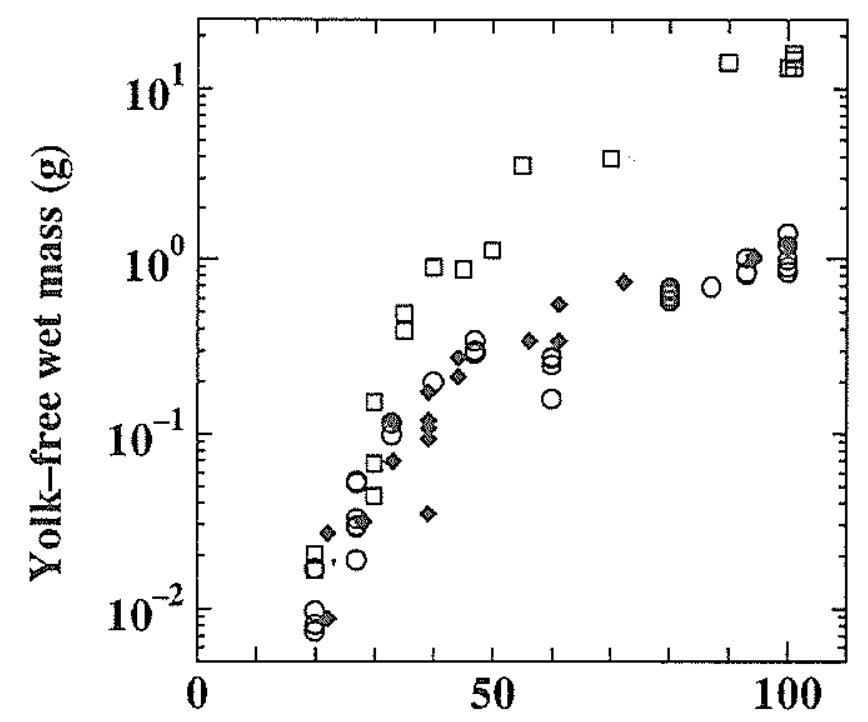

\section{Normalized Incubation}

Fig. 6. Relationships between the logarithm of yolk-free wet mass $(g)$ and normalized incubation time (4) in tits (circles), budgerigar (diamonds), and crows (squares).

decreases after 45-50s of normalized incubation (Fig. 6). The development of MHR in the same altricial species increases at a higher rate during the early phase and then the rate of increase decreases after a phase change at about $40 \%$ of normalized incubation (Fig. 5 ). In contrast, the relationships between MHR and embryo wet mass appear more complex. In common to the species investigated in experiment II, MHR increases in direct proportion to embryo wet mass during the early phase of incubation $(20-40 \%)$ (Fig. 4). However, MHR is independent of embryo $M_{y f}$ in budgerigar and crow embryos after the early phase (i.e. 40-50\% of incubation), coincident with the decrease in embryo growth rates and MHR only increases

\section{REFERENCES}

(1) Akiyama, R., Mitsubayashi, H., Burggren, W.W. and Tazawa, H., Heart rate responses to altered oxygen in early (days 3-9) chick embryos in the intact egg. J. Comp. Physiol. B 169, (1998), p85-92.

(2) Akiyama, R., ono, H., Höchel, J., Pearson, $J . T$, and Tazawa, H., Noninvasive determination of instantaneous heart rate in developing avian embryo by means of acoustocardiogram. Med. \& Biol. Eng. \& Comput. 35, (1997), p323-327.

(3) Ar, A. and Rahn, H., water in the avian egg: overall budget of incubation. Amer. Zool. $20,(1980)$, p373-384.

(4) Ar, A: and Tazawa, H., Analysis of heart rate in developing bird embryos: Effects of mass and developmental mode. Comp. Biochem. physiol. (in press, also this volume), (1999). significantly much later during the final phase. Although the relative growth rates and the rate of increase in MHR in the smaller tit embryos decrease with respect to age at 458 of incubation, MHR continues to increase and is directly proportional to embryo $M_{y f}$ until. 808 of incubation ( $F$ ig. 4). Thus MHR is independent of $\mathrm{M}_{y f}$ at a critical mass of $0.80 \mathrm{~g}$ ( $80 \%$ of hatchling $\left.M_{y f}\right), 0.16 \mathrm{~g}(13 \%)$ and $3.00 \mathrm{~g}(28)$ in the tit, budgerigar and crow, respectively. It is noteworthy that the MHR of the large crow embryo at $40 \%$ of incubation (earlyintermediate phase transition) is already a larger fraction of the hatchling MHR (95\%) than both tit and budgerigar embryos at the same age. We therefore conclude that embryos from smaller eggs, such as the ti.ts, take longer to reach their higher hatchling MHR (with respect to normalized incubation time) as the MHR of pipped-embryos is negatively correlated with fresh egg massinoo rot. "). As a result the development of MHR in most small altricial species is more likely to continualiy increase during all phases of incubation (but not necessarily the same rate of increase) than larger species, such as precocial species due to scaling considerations alone.

\section{ACKNOWLEDGEMENT}

This research was supported in part by a Grant-in-Aid for Encouragement of Young Scientists (awarded to J.T.P. , No. 08750491), a Grant-in-Aid for International collaborative Research (H.T., No. 0704410 ) and a Grant-in-Aid for Scientific Research (H.T.r No. 08650471) from the Ministry of Education, science and Culture (Monbusho).

(5) Bartholomew, G.A. and Goldstein, D.L., The energetics of development in a very laxge altricial bird, the brown pelican. In: R.S. seymour (ed.) Respiration and metabolism of embryonic vertebrates. Dr Junk Publishers. Dordrecht/Boston/London, (1984), p347-357.

(6) Bucher, T.I., Patterns in the massindependent energetics of avian development. J. Experimental zool., Supplement 1, (1987), p139-150.

(7) Bucher, T.L. and Bartholomew, G.A., Analysis of variation in gas exchange, growth patterns, and energy utilization in a parrot and other avian embryos. In: R.S. Seymour (ed.) Respiration and metabolism of embryonic vertebrates. Dr Junk Publishers. Dordrecht/Boston/London, (1984), p359-372.

(B) Burggren, W.W., Tazawa, H. and Thompson, D., Intraspecific variability in avian embryonic heart rate: potential genetic and maternal environmental influences. Isr. J. zool. 40, (1994), p351-362. 
(9) Cain, J.R., Abbott, V.K. and Rogallo, V.I.. Heart rate of the developing chick embryo. Proc. Soc. Exp. Biol. Med. 126, (1967), p507-510.

(10) Dietz, M.W., van Kampen, M., van Griensven, M.J.M. and van Mourik, S., Daily energy budgets of avian embryos: the paradox of the plateau phase in egg metabolic rate. Physiol. Zool. 71, (1998), p147-156.

(11) Moriya, $K$, Höchel, J., Pearson, J.T. and Tazawa, H., Cardiac rhythms in developing chickens. Comp. Biochem. Physiol. (in press, also this volume), (1999).

(12) Pearson, J.T., Development of thermoregulation and posthatching growth in the altricial cockatiel Nymphicus hollandicus. Physiol. Zool. 71, (1998), p237-244.

(13) Pearson, J.T., Tsudzuki, M. , Nakane, X., Akiyama, R. and Tazawa, H., Development of heart rate in the precocial king quail, Coturnix chinensis. J. Exp. Biol. 201, (1998), p931-941.

(14) Ricklefs, R.E. and Starck, J.M., Embryonic growth and development. In: J.M. Starck and R.E. Ricklefs (eds.) Avian growth and development: evolution within the altricial-precocial spectrum . oxford University Press New York/Oxford, (1998), p3 $31-58$.

(15) Sotherland, P.R. and Rahn, $H_{*}$, On the composition of bird eggs. Condor 89 , (1987), p48-65.

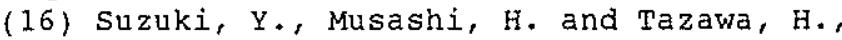
Noninvasive heart rate monitoring system for avian embryos based on ballistocardiogram. Med. Biol. Eng. Comput. 27, (1989), p399-404.

(17) Tazawa, H., Ar, A., Pearson, J.T., Moriya, $K$. and Gefen, $E$., Heart rate in developing ostrich embryos. Brit. Poult. Sci. 39, (1998), p161-166.

(18) Tazawa, H., Hashimoto, Y., Takami, M., Xufu, Y. and whittow, G.C., Simple, noninvasive system for measuring the heart rate of avian embryos and hatchlings by means of a piezoelectric film. Med. Biol. Eng. Comput. $31,(1993)$, p129-134.

(19) Tazawa, H., Hiraguchi, T., Kuroda, O., Tullett, S.G. and Deening, D.C., Enbryonic heart rate during development of domesticated birds. Physiol. Zool. 64, (1991), p1002-1022.
(20) Tazawa, H., Kuroda, o and Whittow, G.C., Non-invasive determination of embryonic heart rate during hatching in the brown noddy (Anous stolidus). Auk 108, (1991), p594-601.

(21) Tazawa, H., Mitsubayashi, H., Hirata, M., Hochel, J. and pearson, J.T., Cardiac rhythms in chick embryos during hatching. Comp. Biochen. Physiol. (in press, also this volume), (1999).

(22) Tazawa, H., Suzuki, Y. and Musashi, H., Simultaneous acquisition of ECG, BCG and blood pressure from chick embryos in the egg. J. Appl. Physiol. 67, (1989), p.478-483.

(23) Tazawa, H., Takami, M., Kobayashi, K., Hasegawa, J. and Ar, A., Non-invasive determination of heart rate in newly hatched chicks. Brit. Poultry sci. 33, (1992), p1111-1118.

(24) Tazawa, H., Watanabe, W. and Burggren, W.W., Embryonic heart rate in altricial birds, the pigeon (Columba domestica) and the bank swallow (Riparia riparia). Physiol. zool. 40, (1994), p1448-1460.

(25) Tazawa, H. and Whittow, G.C., Embryonic heart rate and oxygen pulse in two procellariiform seabirds, Diomedea immutabilis and Puffinus pacificus. J. comp. Physiol. B 163, (1994), p642-648.

(26) Van Mierop, L.H.S. and Bertuch, C.J.JI., Development of axterial blood pressure in the chick embryo. Am. J. Physiol. 212 , (1967), p43-44.

(27) Vleck, C.M. and Bucher, T.L., Energy metabolism, gas exchange, and ventilation. In: J.M. Starck and R.E. Ricklefs (eds.) Avian growth and development: evolution within the altricial-precocial spectrum. oxford university Press New York/oxford, (1998), p89-116.

(28) Vleck, C.M. and Vleck, D., Metabolism and energetics of avian embryos. J. Experimental Zoo1., Supplement 1, (1987), pl11-125.

(29) Wilkinson, I. , SYSTAT: the system for statistics. Evanston, IL: SYSTAT, Inc., (1990), 638pp.

(30) Yeager, D.P. and Ultsch, G.R., Physiological regulation and conformation: A BASIC program for the determination of critical points. Physiol. Z001. 62, (1989), p888-907. 


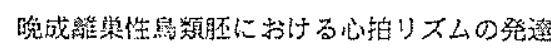

ビーソンジェームズ、田檌皓

\section{概琶}

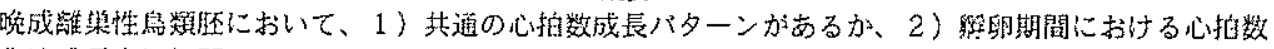

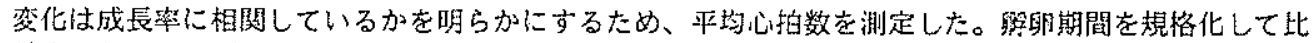

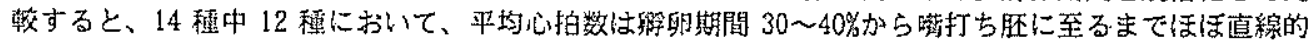

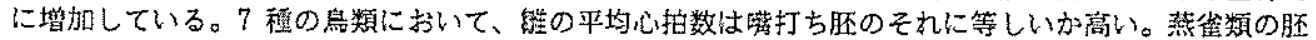

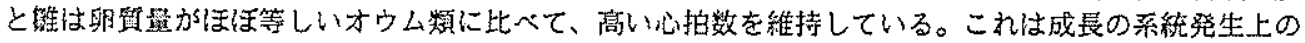
相暹を及映しているものと思われる。肧の平均心拍数は、シシュウカラ、セキセイインコ及びカラスで

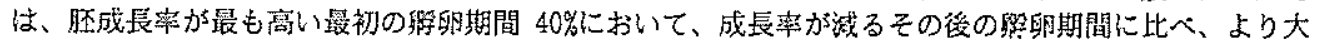

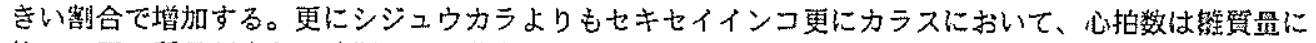

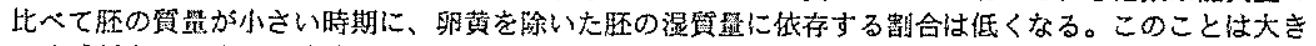

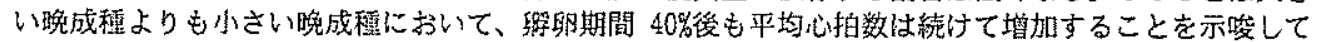
いる。

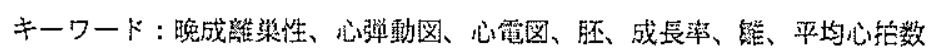

